\title{
Magnetic structure of hexagonal YMnO3 compound: A non- collinear spin DFT study
}

\author{
Lima, A. F.*; Lalic, M. V.
}

*adilmol@gmail.com

The hexagonal $\mathrm{YMnO}_{3}(\mathrm{~h}-\mathrm{YMO})$ is one of the most studied magnetoelectric materials because of its suitability for usage in ferroelectric (FE) memories and due to the intriguing coexistence of ferroelectricity and magnetism. It has a high ferroelectric-paraelectric transition temperature $\left(\mathrm{T}_{\mathrm{c}} \sim 1258 \mathrm{~K}\right)$, and a low antiferromagnetic $(\mathrm{AFM})-$ paramagnetic $(\mathrm{PM})$ transition temperature $\left(\mathrm{T}_{\mathrm{n}} \sim 75 \mathrm{~K}\right)$. For $\mathrm{T}<\mathrm{T}_{\mathrm{n}}$ the $\mathrm{h}$-YMO is simultaneously AFM and FE, exhibiting a clear magnetoeletric characteristic. The magnetism in this compound arises from $\mathrm{Mn}^{3+}$ ions, in $3 \mathrm{~d}^{4}$ configuration, with high spin state, $\mathrm{S}=2$. Despite numerous investigation about of the $\mathrm{h}-\mathrm{YMO}$ magnetic structure, it is still under debate in the literature [1-3]. In this work we performed a non-collinear spin density functional theory (DFT) study in order to obtain the magnetic ground state of the h-YMO compound. The calculations were carried out using a full potential linearized augmented plane wave method as embodied in the Elk computer code. With this computational tool, we could simulate all magnetic configurations described by the experiments for the h-YMO crystal with and without SOC interaction. The lowest energy was found to $\mathrm{P}^{\prime}{ }_{3}$ magnetic structure when the SOC are present. Our results shown that the inclusions of the SOC interaction, in fact, give rise a small FM component along the $\mathrm{c}$ axis in agreement with previous experimental observation [3].

[1] A. Munoz et al., Phys. Rev. B 62, 9498 (2000).

[2] P. J. Brown and T. Chatterji, J. Phys.: Condens. Matter 18, 10085 (2006). [3] K. Singh et al., J. Phys.: Condens. Matter 25, 416002 (2013). 